\title{
Research Paper \\ The Role of Family Emotional Atmosphere, Sense of Coherence, and Affects in the Prediction of Tendency Toward Substance Use Among University Students
}

\author{
Mohammad Arghabaei ${ }^{1} \odot$, *Ali Akbar Soleimanian ${ }^{2} \odot$, Mohammad Mohammadipour $^{3}$
}

1. PhD Student in Consultation, Department of Psychology, Faculty of Humanities, Bojnourd Branch, Islamic Azad University, Bojnourd, Iran. 2. PhD in Consultation, Associate Professor, Department of Educational Sciences, Faculty of Humanities, Bojnourd University, Bojnourd, Iran

3. PhD in Educational Psychology, Assistant Professor, Department of Counseling and Psychology, Quchan Branch, Islamic Azad University, Quchan, Iran.

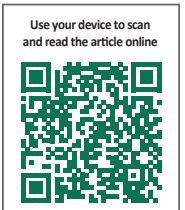

Citation: Arghabaei M, Soleimanian AA, Mohammadipour M. [The Role of Family Emotional Atmosphere, Sense of Coherence, and Affects in the Prediction of Tendency Toward Substance Use Among University Students (Persian)]. Iranian Journal of Psychiatry \& Clinical Psychology. 2018; 24(3):310-323. http://dx.doi.org/10.32598/ijpcp.24.3.310

http://dx.doi.org/10.32598/ijpcp.24.3.310

Funding: See Page 321 (c) Copyright: The Author(s)

Received: 22 Feb 2018 Accepted: 12 Jun 2018

Keywords:

Family atmosphere, Sense of coherence, Affects, Substance use, University students

\begin{abstract}
A B S TRACT
Objectives Tendency toward substance use is influenced by a range of individual, familial, and social factors. This study aimed to investigate the relationship between family emotional atmosphere, sense of coherence, and affects with tendency toward substance use among university students. Methods In this descriptive-correlative study, a total of 400 students (311 males and 89 females) were selected using multistage cluster sampling method from Shahid Beheshti Teacher Training University, Mashhad, Iran. Then they completed measures of parental-child interaction rating scale, sense of coherence scale, positive and negative affect scale, and tendency toward addiction scale. Data analysis was conducted using Pearson correlation and backward multiple regression.

Results There were significant and negative relationships between subscales of family emotional atmosphere, sense of coherence, and positive affects with tendency toward substance use among students $(P<0.01)$. Moreover, negative affects were positively correlated with tendency toward substance use $(r=0.48 ; P<0.01)$. Regression analysis also indicated that a positive family emotional atmosphere and sense of coherence were significant negative predictors of tendency toward substance use whereas negative affects were significant positive predictors. However, the role of positive affects in the prediction of tendency toward substance use was not significant.

Conclusion Addressing family problems, developing the sense of individual coherence, and instructing strategies to regulate negative emotions in prevention and intervention programs may help reduce tendency toward substance use among university students.
\end{abstract}

\section{Extended Abstract}

\section{Introduction}

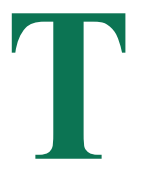

he prevalence of substance use increases from $1.3 \%$ at age 12 to $25.4 \%$ at age 21 , and people aged 18-24 years have the highest rate of substance use [5]. This age coincides with students' admission to universities. Thus, university students are a high-risk group for substance use. Etiological studies have shown that substance use is influenced by a range of bio-psycho-social factors [6]. Family emotional atmosphere is a familial factor associated with the youths' substance use [7], with studies indicating that poor family relationships may facilitate tendency toward substance use [9].

Sense of coherence is also an individual factor which may influence substance use. Existing literature shows that great coherence is a protective factor against psychopathology and

\section{* Corresponding Author:}

Ali Akbar Soleimanian, PhD

Address: Department of Educational Sciences, Faculty of Humanities, Bojnourd University, Bojnourd, Iran.

Tel: +98 (945) 3155111

E-mail: soliemanian@gmail.com 
decreases the risk of psychological disorders [14]. Although there is little evidence for the relationship between sense of coherence and substance use, few studies have indicated that a higher sense of coherence may cause lower tobacco use, alcohol use, and drug use [18, 19]. Finally, affects have also suggested as a psychological factor contributing to substance use. That is, those who experience more negative affects are at a higher risk of substance use [22].

Despite these studies, little attention has been paid to tendency toward substance use and there is a lack of sufficient evidence for the relationship between sense of coherence and affects (especially positive affects) with substance use. Accordingly, the aim of this study was to examine the relationship between family emotional atmosphere, sense of coherence, and affects with tendency toward substance use among university students.

\section{Method}

The statistical population of this descriptive-correlative study included all male and female students studying in the academic year of 2017-2018 in the Shahid Beheshti Teacher Training University, Mashhad, Iran. In this study, a multistage cluster sampling method was applied to recruit 400 students (including 311 males and 89 females) aged 18-56 years old $(\mathrm{M}=24.20 ; \mathrm{SD}=8.07)$. To do so, of the total $11 \mathrm{ma}-$ jors in Shahid Beheshti Teacher Training University, six ma- jors (including mathematics, chemistry, educational science, psychology of exceptional children, counseling, and Persian literature) were selected randomly; from each major, several classes were selected, and in each class, several students were selected to answer the measures. For data collection, a set of measures was use, including Parental-Child Interactions Rating Scale, Sense of Coherence Scale, Positive and Negative Affect Scale, and Tendency toward Substance Use Scale. Examining psychometric properties of all these measures has shown their satisfactory reliability and validity in Iranian samples.

To analyze the data and examine the relationship between variables, a Person correlation was conducted. Moreover, a multivariate backward regression analysis was conducted to examine the role of independent variables (including family emotional atmosphere, sense of coherence, and affects) in the prediction of tendency toward substance use.

\section{Results}

As shown in Table 1, there are significant relationships between all variables and tendency toward substance use, with manageability $(r=-0.56)$ and shared experiences $(r=-$ 0.19 ) having the strongest and the weakest relationships with students' tendency toward substance use, respectively. Referring to Table 2, regression analysis indicated that except "'affection", "Caress", and 'Positive Affects",

Table 1. The relationship between family atmosphere, coherence, and affects with students' tendency toward substance use

\begin{tabular}{ccccc}
\hline Variables & Sub-Scales & M & SD & Tendency Toward Substance Use \\
\hline \multirow{2}{*}{ Comprehensibility } & 42.27 & 7.91 & $-0.42^{* *}$ \\
& Meaningfulness & 37.99 & 9.21 & $-0.51^{* *}$ \\
Manageability & 43.89 & 9.39 & $-0.56^{* *}$ \\
Affection & 7.74 & 1.79 & $-0.40^{* *}$ \\
Caress & Approval & 6.66 & 2.07 & $-0.26^{* *}$ \\
Samily atmosphere & Shared experiences & 6.96 & 1.82 & $-0.30^{* *}$ \\
& Giving gifts & 6.06 & 1.98 & $-0.19^{* *}$ \\
& Encouragement & 6.02 & 2.21 & $-0.34^{* *}$ \\
& Trust & 7.11 & 1.95 & $-0.39^{* *}$ \\
& Sense of security & 7.39 & 1.95 & $-0.43^{* *}$ \\
& Negative affects & 7.72 & 1.97 & $-0.44^{* *}$ \\
\hline Affects & Positive affects & 23.05 & 8.44 & $0.48^{* *}$ \\
& & 33.64 & 6.78 & $-0.37^{* *}$ \\
\hline
\end{tabular}


Table 2. Multivariate regression analysis with backward method for students' tendency towards smoking

\begin{tabular}{|c|c|c|c|c|c|c|c|c|c|}
\hline Predictors & B & $\beta$ & $\mathbf{t}$ & $\mathbf{P}$ & $\mathbf{R}$ & $\mathbf{R}^{2}$ & $\mathbf{F}$ & df & $\mathbf{P}$ \\
\hline Comprehensibility & -0.083 & -0.077 & -2.09 & $<0.05$ & \multirow{10}{*}{0.691} & \multirow{10}{*}{0.477} & \multirow{10}{*}{31.12} & \multirow{10}{*}{$10-341$} & \multirow{10}{*}{$<0.001$} \\
\hline Meaningfulness & -0.152 & -0.167 & -2.79 & $<0.01$ & & & & & \\
\hline Manageability & -0.167 & -0.184 & -2.62 & $<0.01$ & & & & & \\
\hline Approval & -0.456 & -0.096 & -1.65 & $<0.01$ & & & & & \\
\hline Shared experiences & -0.635 & -0.146 & -2.66 & $<0.01$ & & & & & \\
\hline Giving gifts & -0.443 & -0.113 & -2.01 & $<0.05$ & & & & & \\
\hline Encouragement & -0.645 & -0.143 & -2.27 & $<0.05$ & & & & & \\
\hline Trust & -0.664 & -0.148 & -2.34 & $<0.05$ & & & & & \\
\hline Sense of security & -0.690 & -0.154 & -2.40 & $<0.05$ & & & & & \\
\hline Negative affects & 0.164 & 0.160 & 3.02 & $<0.01$ & & & & & \\
\hline
\end{tabular}

Iranian Journal of
PSYCHIATRY AND CUINICAL PSYCHOLOGY

others variables were significant predictors of tendency toward substance use, with these variables accounting for $47.47 \%$ of the total variance.

\section{Discussion}

Current results indicated that a higher level of satisfactory family atmosphere was a significant predictor of students' lower tendency toward substance use. According to Risk and Protective Factors Model, conflicts in families and dysfunctional family relationships act as risk factors and increase youths' tendency toward substance use. Indeed, in such circumstances, youths choose substance use as a way to escape from unsatisfactory family conditions [35]. Moreover, lower levels of family coherence and close emotional relationships may cause family borders and parents' supervisory role to disappear, which, in turn, increase children's tendency toward substance use.

In line with previous studies $[18,36]$, these results also showed a significant relationship between sense of coherence and students' tendency toward substance use. High levels of sense of coherence, which gives meaning to everyday life activities, is associated with using efficient coping strategies [36]. This causes those people with high sense of coherence to use effective coping strategies to deal with stressful situations and tend less toward health-threatening behavior such as smoking, alcohol consumption, and drug use [19].

Finally, these results indicated significant role of negative affects in the prediction of tendency toward substance use. The process through which negative affects can increase the likelihood of substance use has been conceptualized as
Negative Affects-Stress Model. According to this model, stressful events may increase levels of negative affects which causes the youths to use drugs as a coping strategy to alleviate adverse affects cause by stressful conditions [38, 39]. Considering current findings, addressing family problems, improving sense of coherence, and developing coping strategies to deal with negative affects may help reduce tendency toward substance use among university students.

\section{Ethical Considerations}

\section{Compliance with ethical guidelines}

The participants signed the informed consent form and had the right to leave the study at any time.

\section{Funding}

This research did not receive any specific grant from funding agencies in the public, commercial, or not-for-profit sectors. The paper was extracted from the first author $\mathrm{PhD}$ thesis, Faculty of Humanities, Bojnourd University.

\section{Conflict of Interest}

The authors declared no conflict of interest.

\section{Acknowledgements}

In the end, we thank all the participants who helped us complete this study. 


\title{
نقش جوّ عاطفى خانواده، احساس انسجام و عواطف در ييشبينى كَرايش به مصرف مواد در دانشجويان

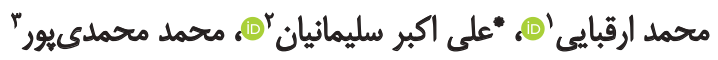

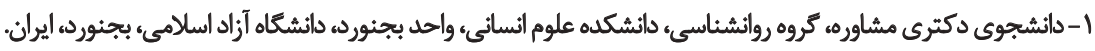

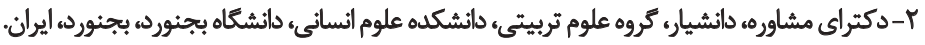

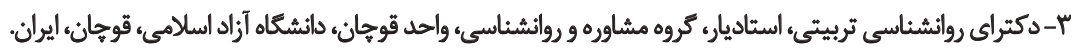

\begin{abstract}
حكيد

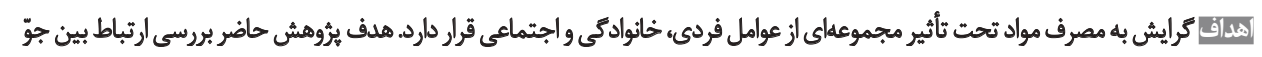

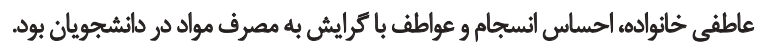

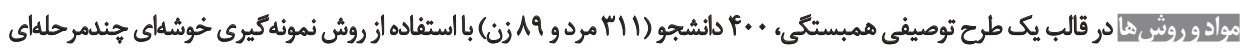

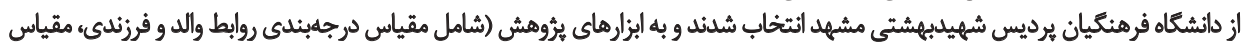

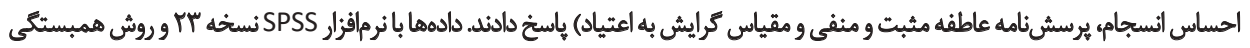

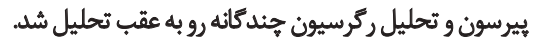

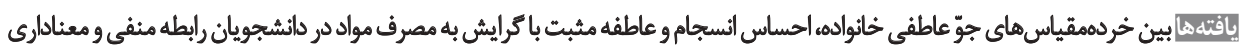

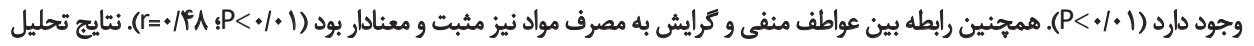

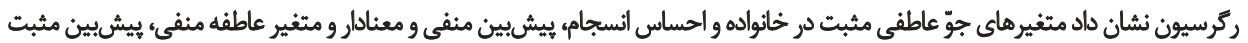

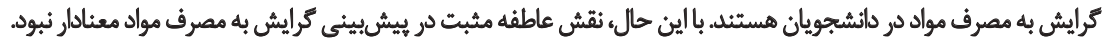

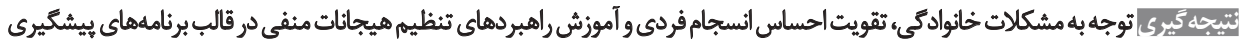

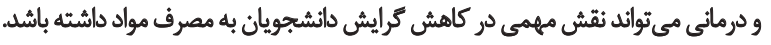

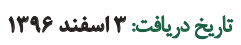

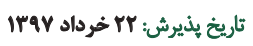

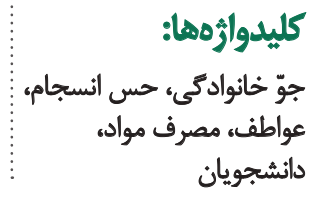

يزشكى، مصرف مواد كماكمان يكى از بزركترين مشكلات أنات

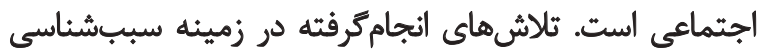

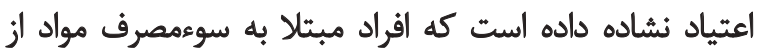

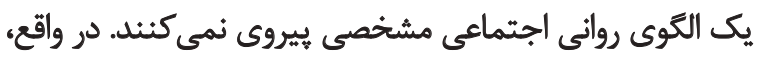

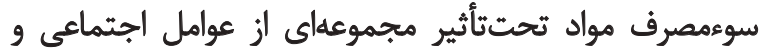

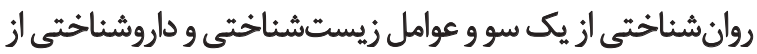

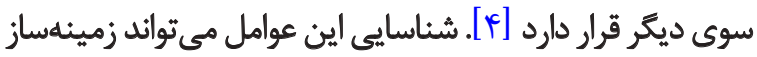

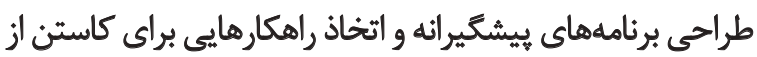

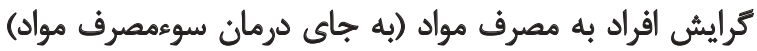

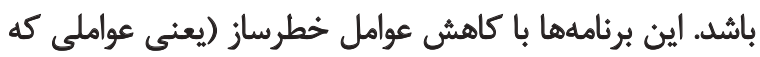

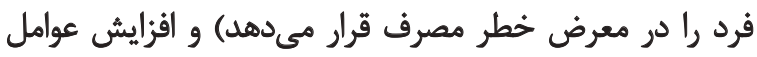

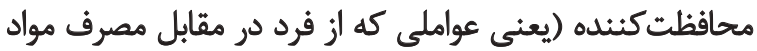

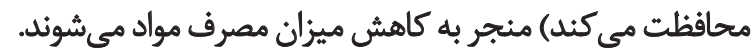

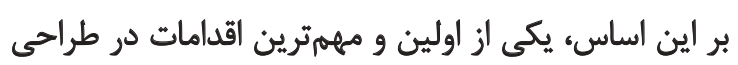

\section{daleo}

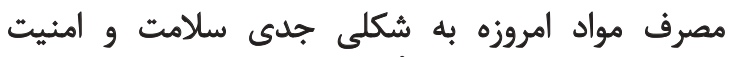

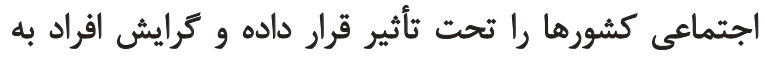

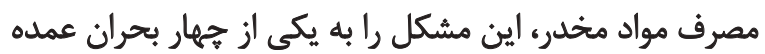

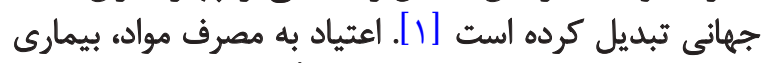

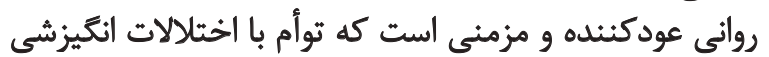

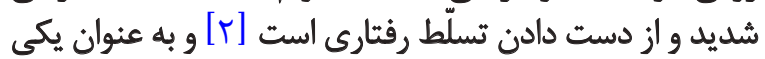

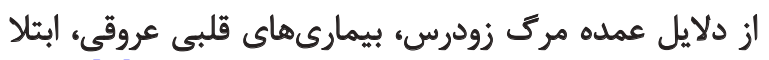

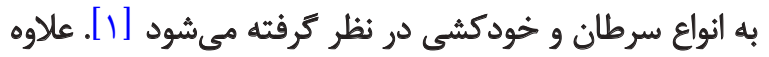

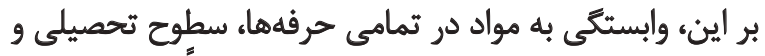

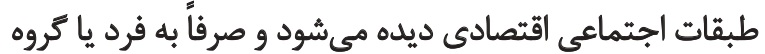

خاصى مربوط نمى طود إنقات باوجود جندين دهه تلاش اجتماعى، حقوقى و ارائه راهكارهاى نـي 
مواد است كه در دسته عوامل روانشئاختى قرار مى كيرد و

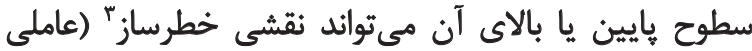

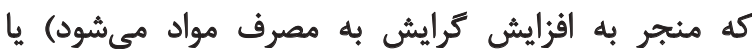

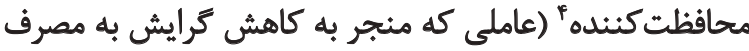

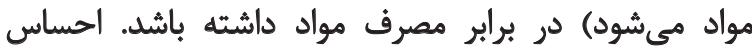

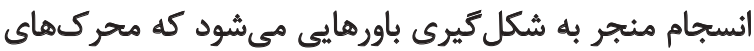

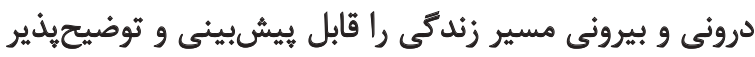

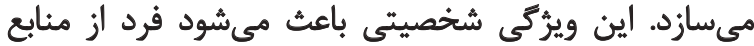

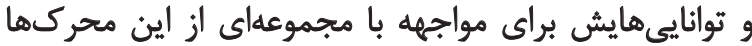

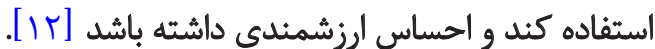

حس انسجام، منبع مقاومت تعميميافتهاى است كه اثرات

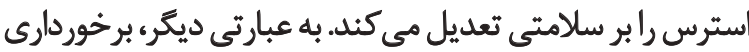

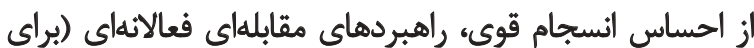

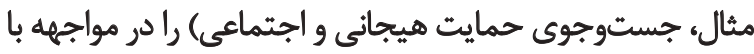

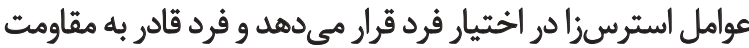

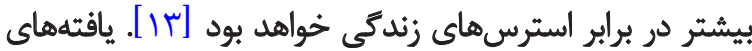

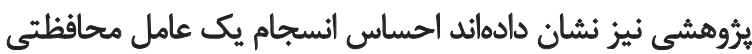

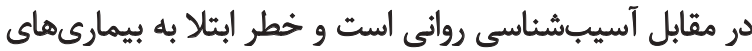
روانى را كاهش مى دهد [If]

بنابراين، افرادى كه از سطوح بالاى اين سازه شخصيتى

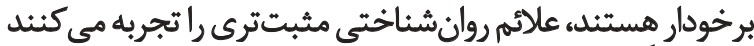

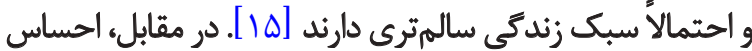

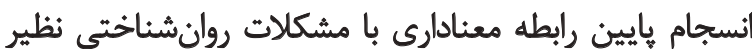

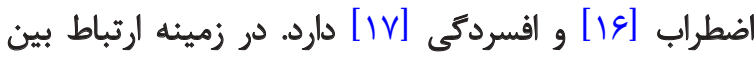

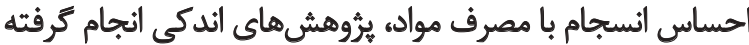

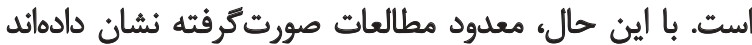

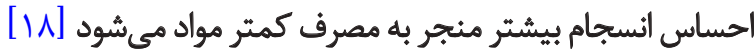

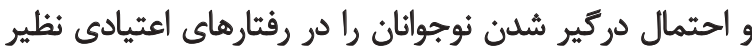

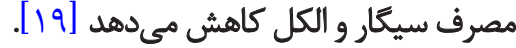

در نهايت، عواطف نيز به عنوان يكى ديكر از متغيرهاى

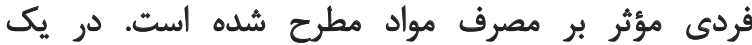

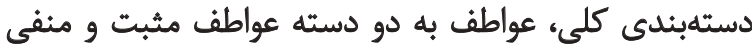

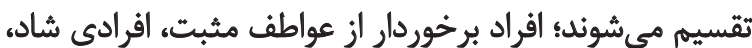

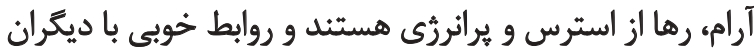

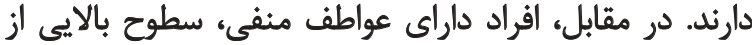

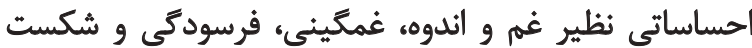

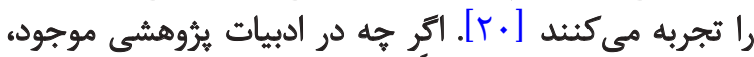

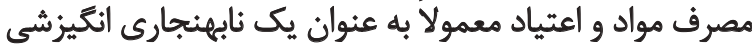

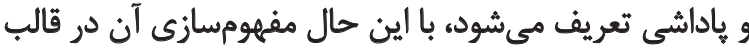
يك اختلال عاطفى نيز ييشنهاد شده است

3. Risk factor

4. Protective factor
برنامه هاى بيشيُيرى از سوءمصرف مواده شناخت اين عوامل

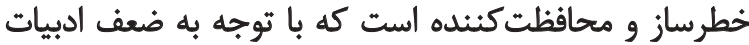

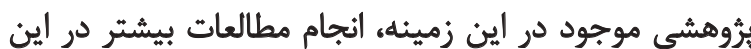
حوزه ضرورى است.

مطالعات همه كيرشناسى نشان مي دهند مصرف مواد در دوران

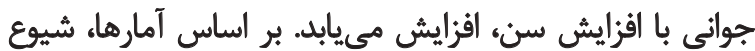

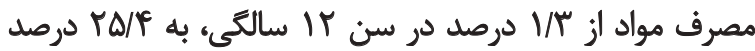

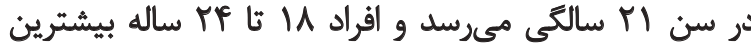

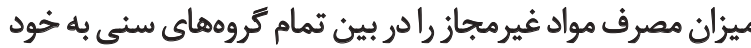

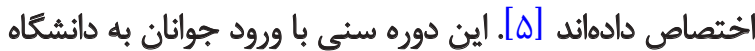

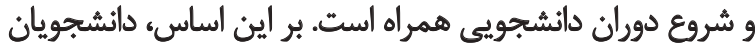

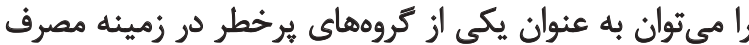

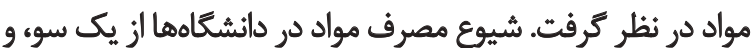

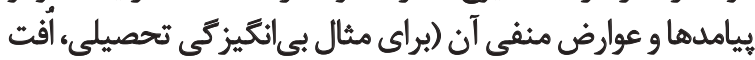

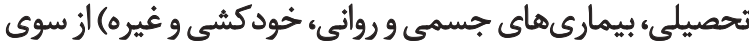

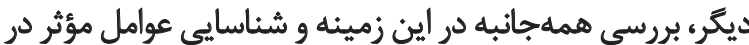

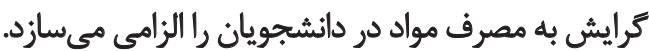
همان طور كه اشاره شد، مطالعات سببشناسي صورت كرفته در جمعيت عمومى نشان دادهاند مصرف مواد متأثر از تعامل بيتيجيده

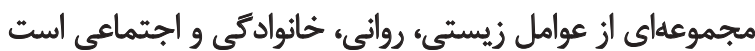

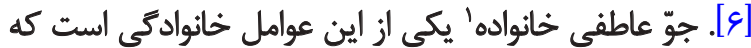

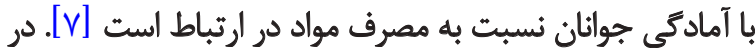

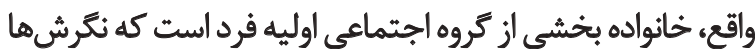

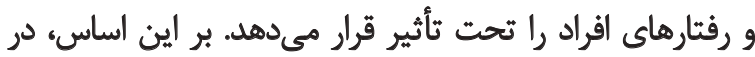

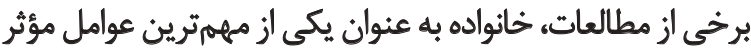

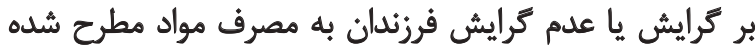

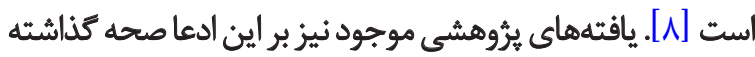

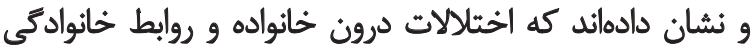

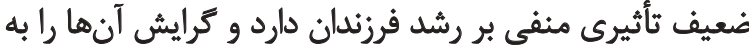

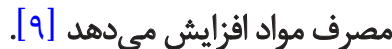
نتايج برخى مطالعات ديگر نشان دادهاند ثفاوت معنادارى بين

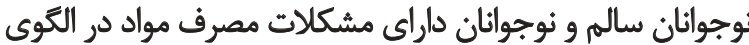

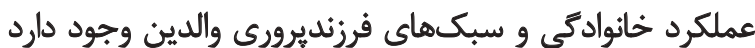

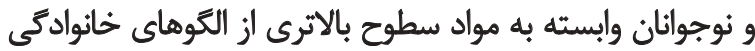

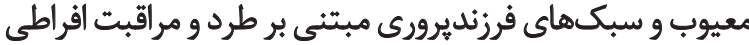

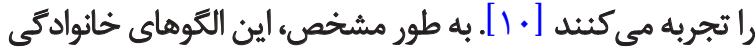

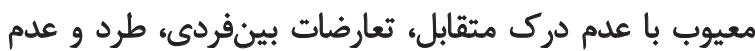

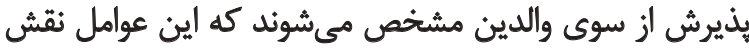

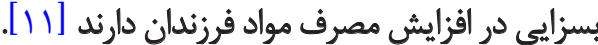

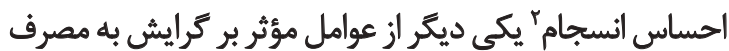

1. Family emotional atmosphere

2. Sense of coherence 
بين احساس انسجام و كرايش به مصرف مواد در دانشجويان

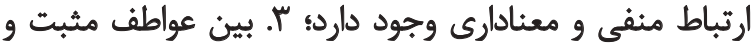

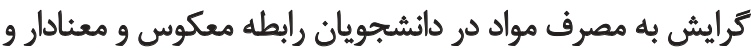

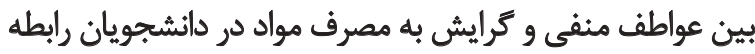

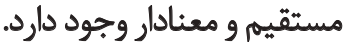

\section{شر كت كثند كان و طرح بيروهش}

نيروهش حاضر از نظر هدف در دسته طرحهاى بنيادى و از

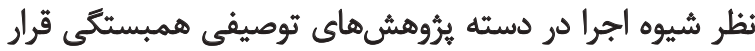

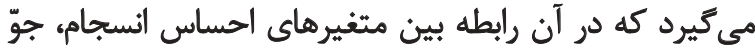

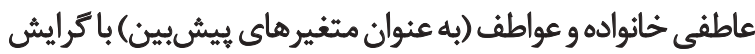

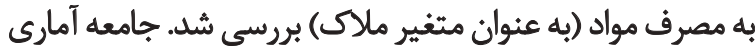

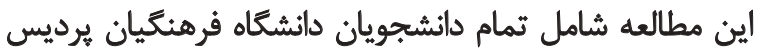

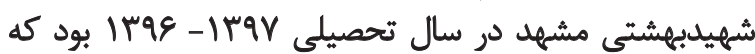

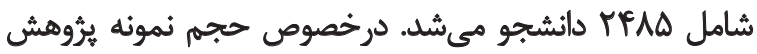

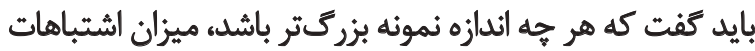

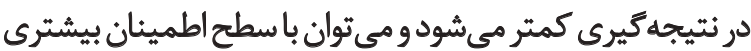

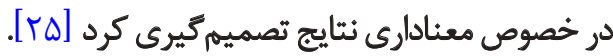

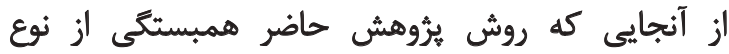

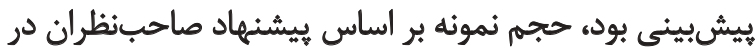

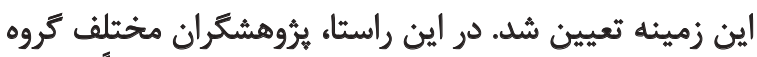

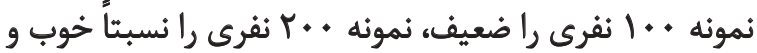

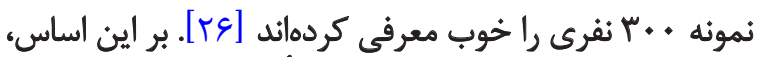

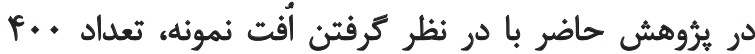

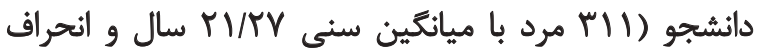

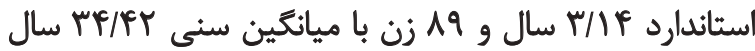

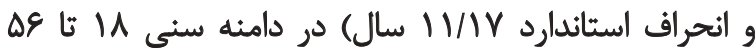

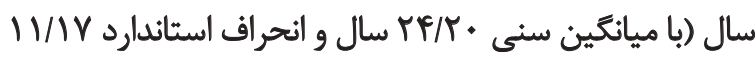

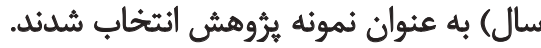

انتخاب نمونه با استفاده از روش نمونهكيرى خوشايى

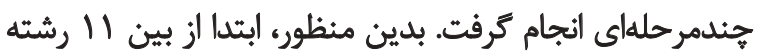

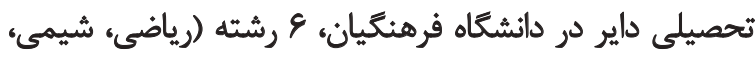

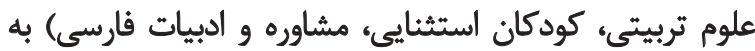

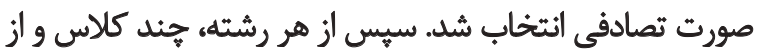

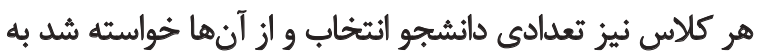

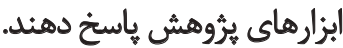

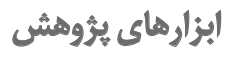

\section{مقياس درجهبندى روابط والد فرزندى}

به منظور ارزيابى جوّ عاطفى خانواده در مطالعه حاضر از مقياس
مطابق با اين مفهومسازى، بىنظمى عاطفى كليد زيربنايى

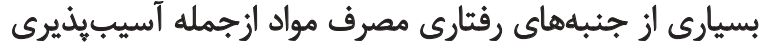

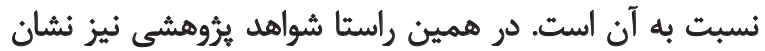

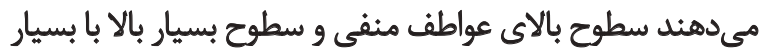

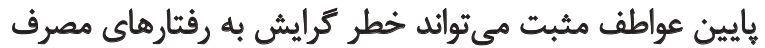

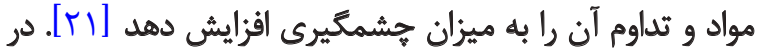

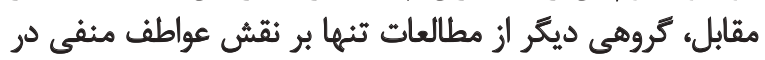

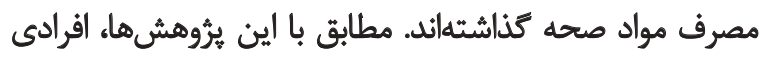

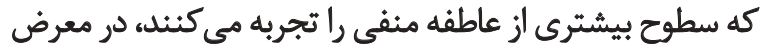

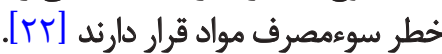

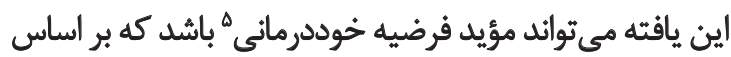

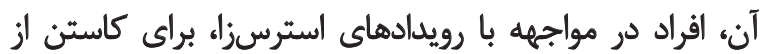

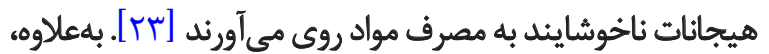

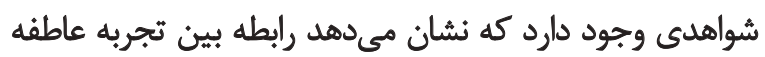

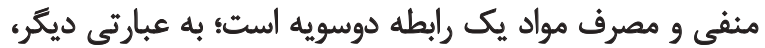

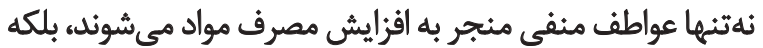

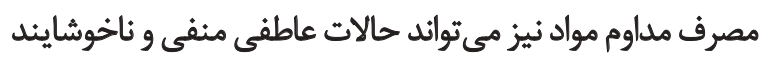

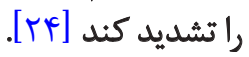

در مجموع، نتايج مطالعات فوق حاكى از ارتباط مجموعهاى از باز

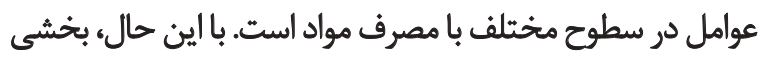

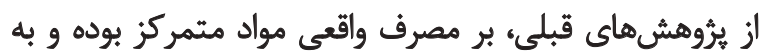

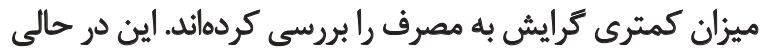

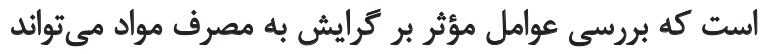

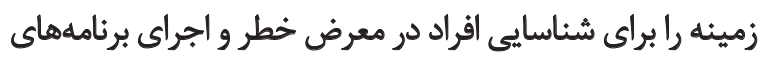

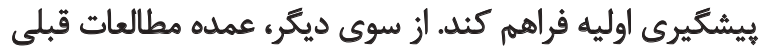

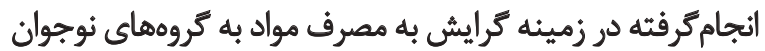

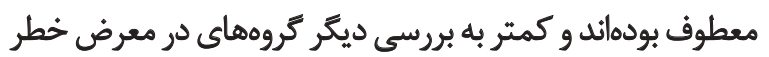

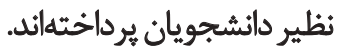

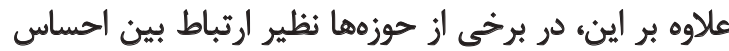

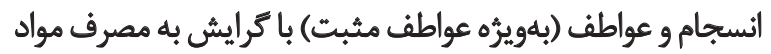

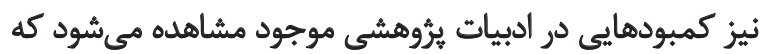

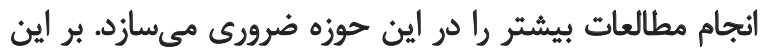

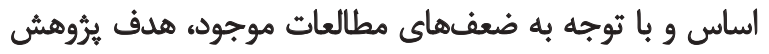

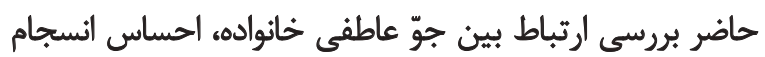

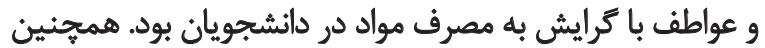

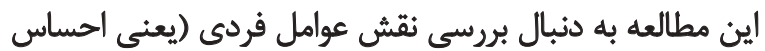

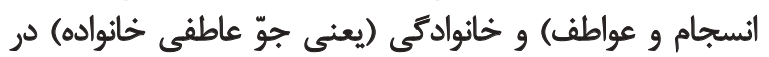
بيشبينى ترايش به مصرف مواد در دانشجويان بود. بر اساس ادبيات برؤوهشى موجود، فرضيههاي اين مطالعه

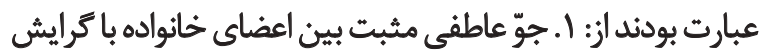

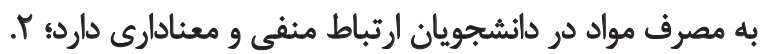




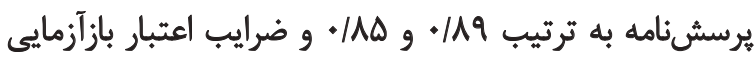

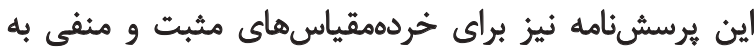

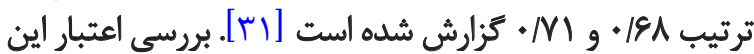

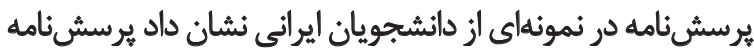

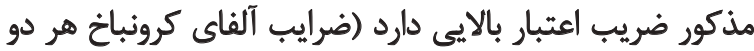

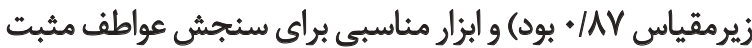

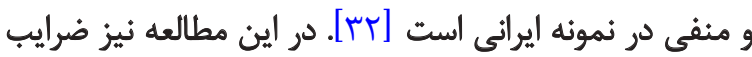

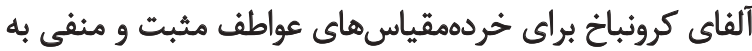

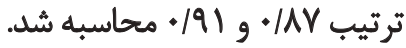

\section{مقياس كرايش بـ اعتياد}

اين مقياس ابزارى 19 سؤالى است كه با هدف بروسي ميزان

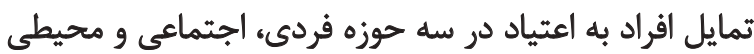

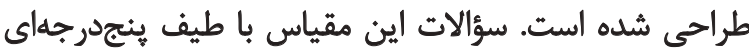

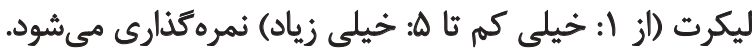

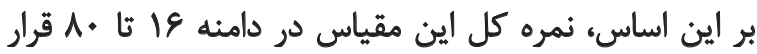

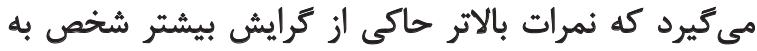

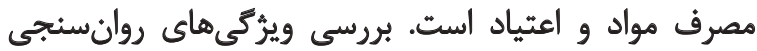

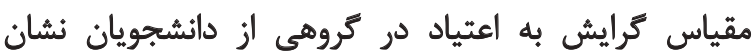

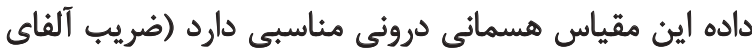

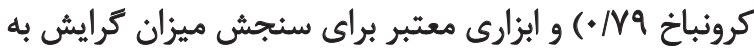

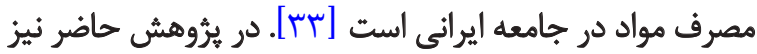

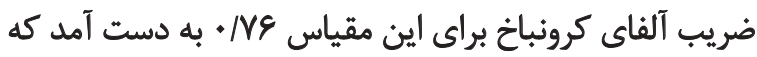
حاكى از اعتبار رضايتبحش كروناخ آن است.

$$
\text { شيوها اجرا }
$$

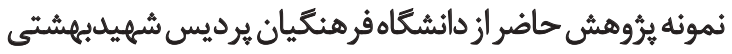

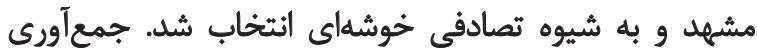

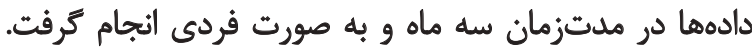

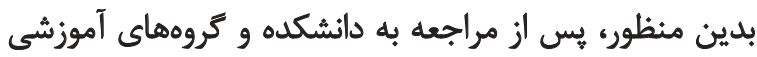

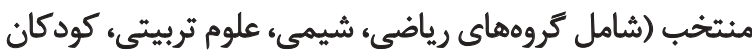

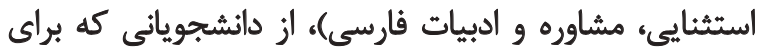

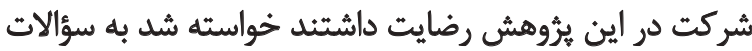

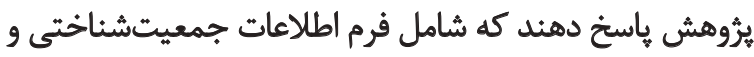

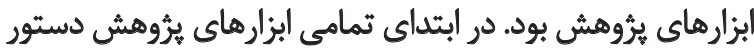

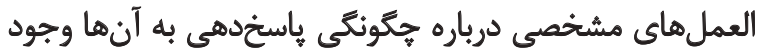

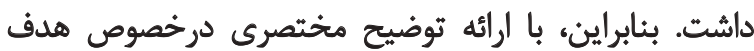

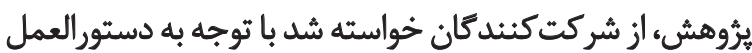

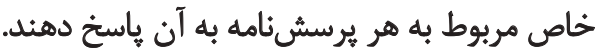

با توجه به حساسيت شركت كنيندكان نسبت به موضوع اين

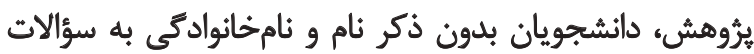

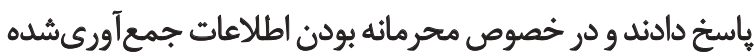

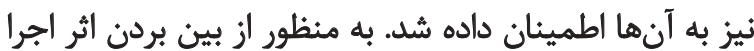

در جهبندى روابط والد فرزندى \& استفاده شد. اين مقياس شامل ناميل

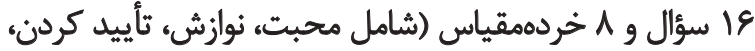

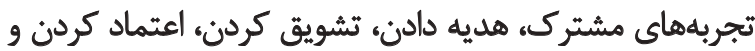

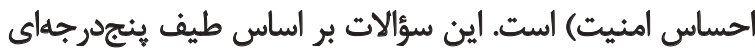

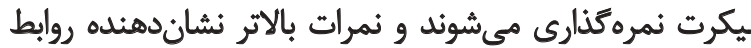

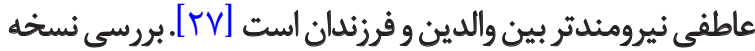

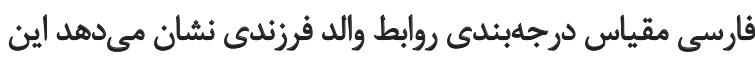

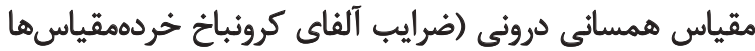

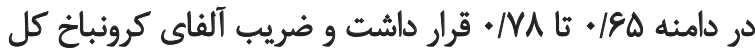

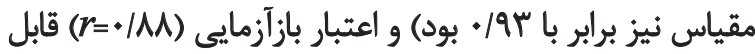

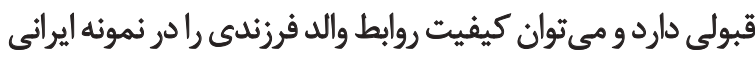

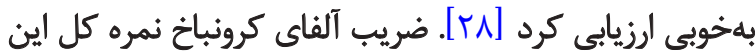

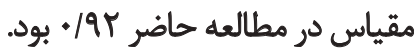

\section{مقياس احساس انسجام}

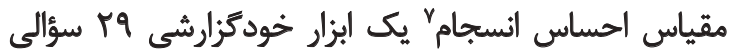

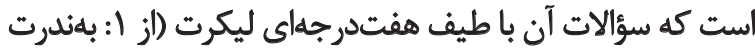

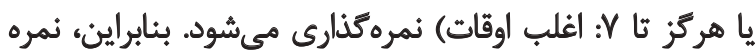

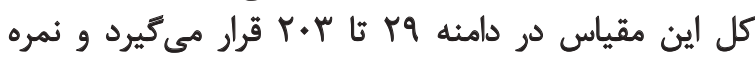

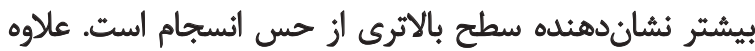

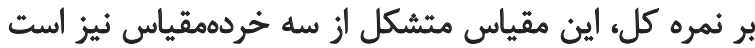

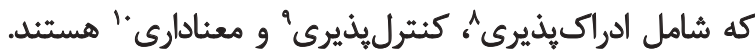

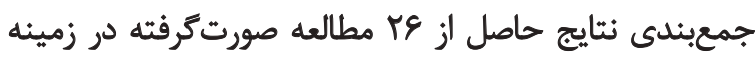

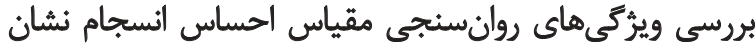

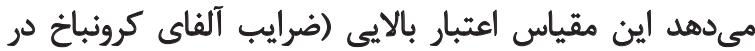

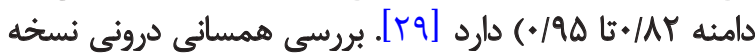

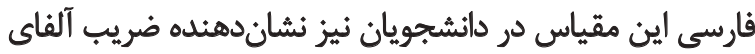

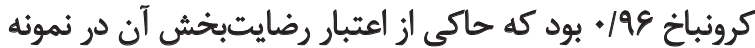

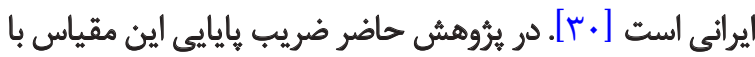

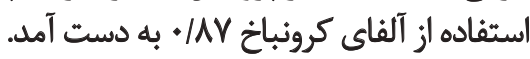

$$
\text { برسش نامه عاطفه مثبت و منفى }
$$

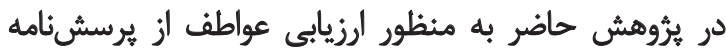

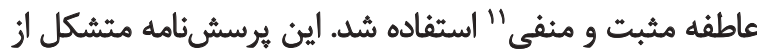

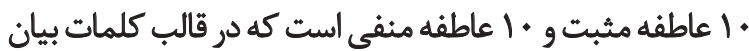

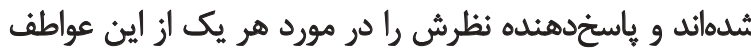

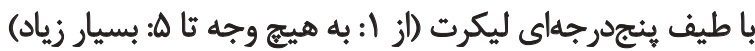

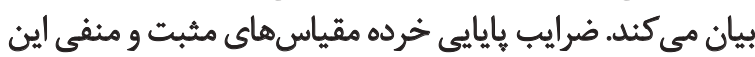

6. Parental-Child Interactions Rating Scale

7. Sense of Coherence Scale (SOC)

8. Comprehensibility

9. Manageability

10. Meaningfulness

11. Positive and Negative Affect Scale (PANAS) 
جدول ا. رابطه بين احساس انسجام، جوّ عاطفى خانواده و عواطف با ترايش به مصرف مواد در دانشجويان

\begin{tabular}{|c|c|c|c|c|}
\hline كرايش به مصرف مواد• & انحراف استاندارد & مياتكين & خردمقياسها & مثغيرها \\
\hline.$- / 4 t$ & V/9I & $r H / T V$ & ادراكيذيرى & \multirow{3}{*}{ حس انسجام } \\
\hline.$- \mid 101$ & $9 / M$ & rV/qq & معنادارى & \\
\hline-.108 & $q / 7 q$ & $e r p / A q$ & كثترل ئيرى & \\
\hline$-+/ 4$ & $1 / 19$ & $P / M^{e}$ & هحبت & \multirow{8}{*}{ جوّ عاطفى خانواده } \\
\hline.$- / 48$ & $r / \cdot \gamma$ & 9l8s & 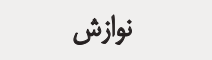 & \\
\hline.$- / m$ & V/AY & e/Q & تأييد كردن & \\
\hline-+119 & $1 / 4$ & 91.8 & تجربه هاي مشترك & \\
\hline$-\cdot / M$ & $T / T$ & gl.r & هليه دادن & \\
\hline$-+/ 49$ & $1 / 9 \Delta$ & $\mathrm{V} / \mathrm{I}$ & تشويق كردن & \\
\hline.$- / 4 \pi$ & $1 / 9 \Delta$ & $V /$ Yq & اعتماد كرنن & \\
\hline$-\cdot / \mu t$ & V/aY & $V / V T$ & احساس امثيت & \\
\hline.$/ p a$ & Nere & $M \% / \cdot \Delta$ & عواطفي منفقى & \multirow{2}{*}{ عواطف } \\
\hline$-\cdot / T r$ & $8 / \mathrm{MA}$ & $M T / g T^{e}$ & عواطف هئبت & \\
\hline
\end{tabular}

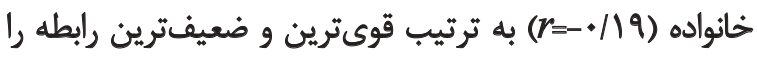

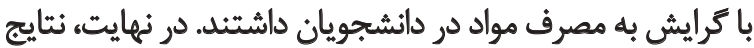

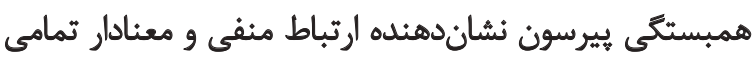

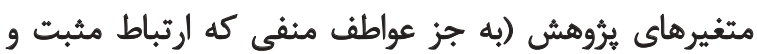

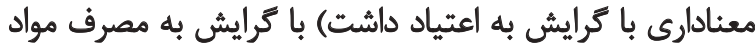

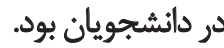

در ادامه، به منظور بررسى نقش احساس انسجام، جوّ عاطفى دوانى

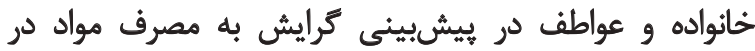

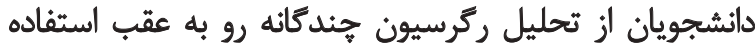

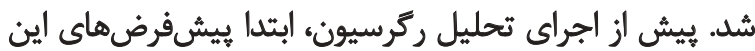

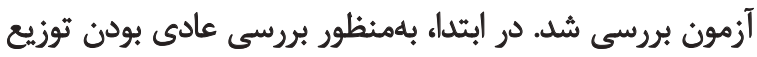

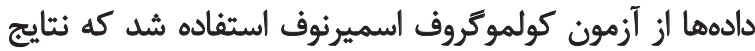

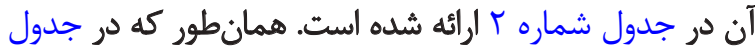

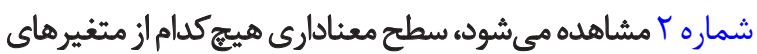

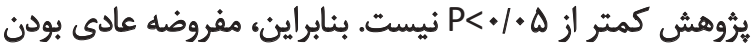
براى تمامى متغيرهاى ثيروهش برقرار است.

در ادامه، به منظور بررسي استقلال خطاها از آزمون دوربين

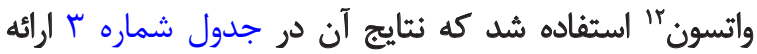

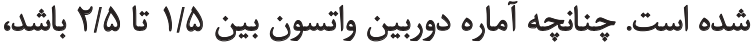

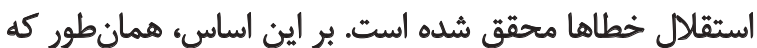

12. Durbin-Watson
نيز ترتيب ارائه ابزارهاى يروهش براى هر يك از دانشجويان به صورت تصادفى تغيير داده شد. به اين صورت كه ايتدا بركائه

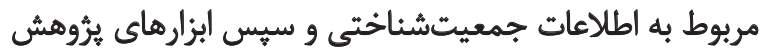

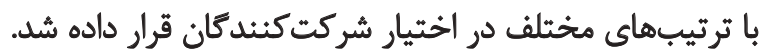

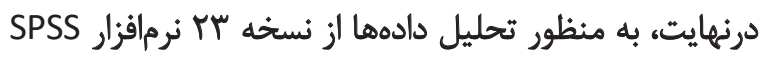

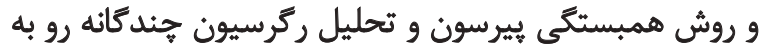
عقب استفاده شد.

batọ

به منظور بررسي ارتباط بين احساس انسجام، جوّ عاطفي

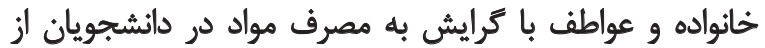

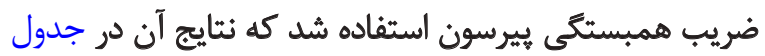

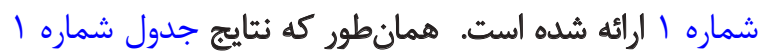

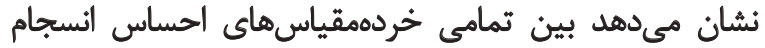

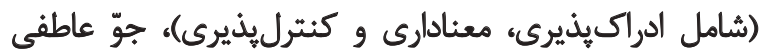

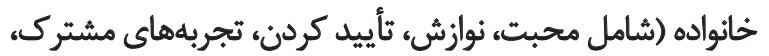

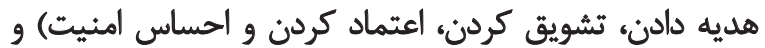

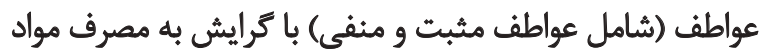

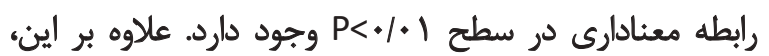

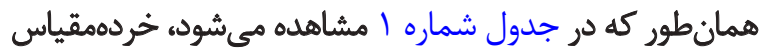

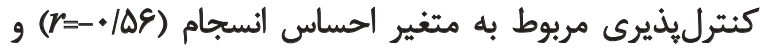

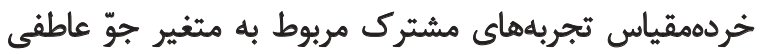


جدول r. نتايج آزمون كولموكروف اسميرنوف به منظور بررسى عادى بودن دادهها

\begin{tabular}{|c|c|c|}
\hline سطح معنادارى & مقدار Z كولموكّروف اسميرنوف & مثغيرها \\
\hline /NTr & $+/ 191$ & ادراكيذيرى \\
\hline.$M I V$ &.$M A$ & 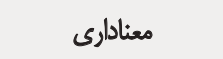 \\
\hline.$/ r$ &.$/ 1 V$ & كتبرلي يذيرى \\
\hline.$/ 110$ &.$/ 1 V^{\circ}$ & محبت \\
\hline.$/ 1 \cdot 9$ &.$/$ VVa & ن موازش \\
\hline . /ar &.$M$ & تأييد كردن \\
\hline.$M I r$ &.$/ 198$ & تجربdهاي مشترى \\
\hline$\cdot / 1 \cdot \Delta$ &.$/ \mathrm{hr}$ & هلهيه دادن \\
\hline.$M H$ & $+/ 191$ & تشويق كردن \\
\hline$\cdot M N$ & /NTr & 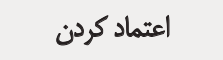 \\
\hline$\cdot M \cdot 4$ &.$/ 1 V 8$ & احساس امئيت \\
\hline.$M A N$ &.$/ 1 a P^{\circ}$ & عواطف منفى \\
\hline.$/ 194$ &.$/ 19 r$ & عواطف هثبت \\
\hline.$/ 19$ &.$/ 19$. & كرايش به هصرف مواد \\
\hline
\end{tabular}

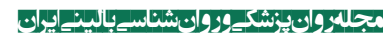

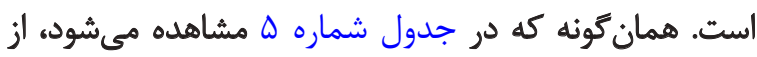

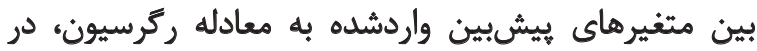

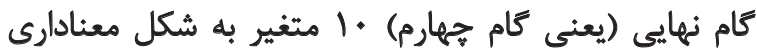

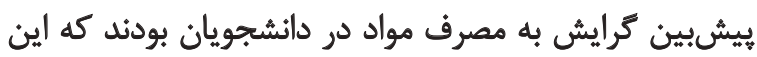

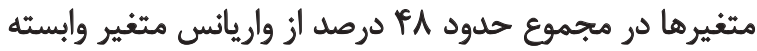

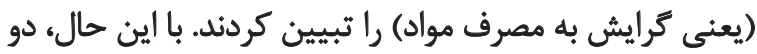

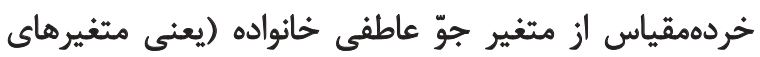

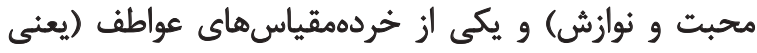

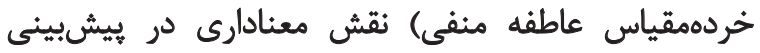

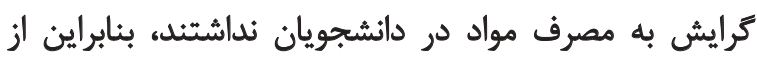
معادله ركرسيون كنار كذاشته شران داندئ. همان كونه كه در جدول شماره 9 مشاهده ميشوده، متغيرهاى

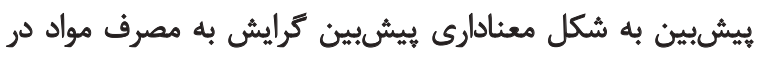

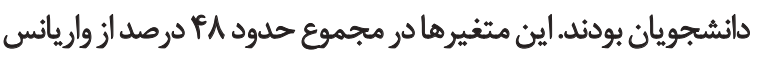

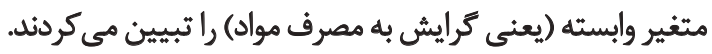

نتايج بررسى اين مفروضه در جدول شماره ب نشان مى نهارده،

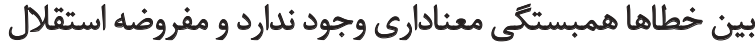

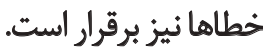

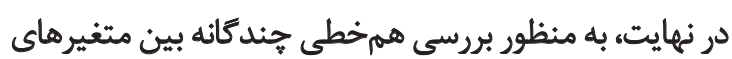

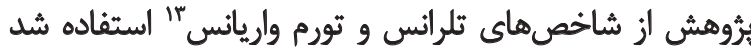

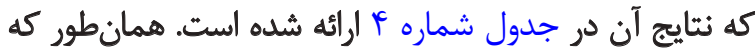

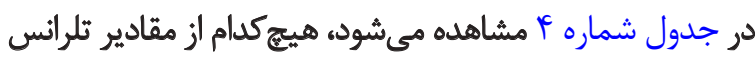

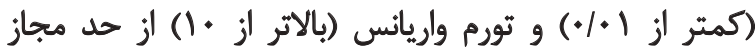

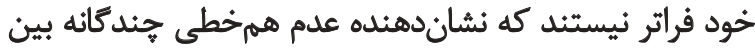
متغيرهاى ثرُوهش است. بنابراين اين مفروضه برقان فرار است.

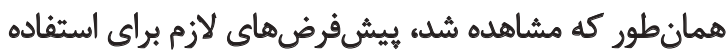

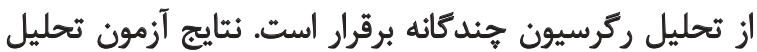

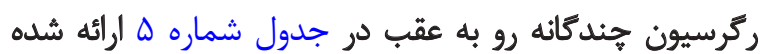

13. Variance Inflation Factor (VIF)

جدول ץ. نتايج آزمون دوربين وائسون به منظور بررسى استقلال خطاها

\begin{tabular}{|c|c|c|}
\hline يذيرش/رد مفروضه & شاخص دوربين واتسون & متغير ملاكى \\
\hline لخئيرش & Wete & كرايش به مصرف مواد \\
\hline
\end{tabular}


جدول f. نتايج آزمون همخطى متغيرها

\begin{tabular}{|c|c|c|}
\hline تورم واريانس & تل تلرانس & متغير \\
\hline $1 / m$ & $\cdot M^{e}$ & الدراكئيرى \\
\hline I/Ta & .14 . & معنادارى \\
\hline $1 / .8$ &.$/ 9 \Delta$ & كثترليذيرى \\
\hline $1 / m e$ & $\cdot M^{f}$ & متبت \\
\hline I/PY & .180 & نوازش \\
\hline $1 / \% 9$ &.$/ V$ & تأييد كردن \\
\hline $1 / 1$ & $+/ 9$ & تجربلهاى مشترى \\
\hline $1 / 1 \%$ & $\cdot / M$ & هديه دادن \\
\hline $1 / F A$ & . IAT & تشويق كردن \\
\hline $1 / \Delta T^{N}$ & .109 & اعتماد كردن \\
\hline Ifer & $\cdot / \Delta \Delta$ & احساس امنيت \\
\hline $1 / 88$ & .181 & عواطف منفى \\
\hline $1 / 19$ & - /Ar & عواطف مثبت \\
\hline
\end{tabular}

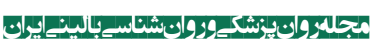

و اجتماعى است كه شناسايى اين عوامل مىتوائد زمينهساز

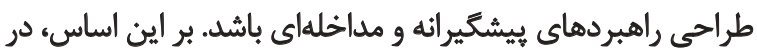

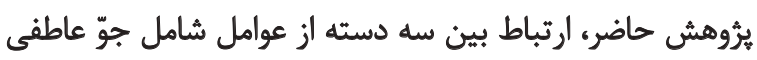

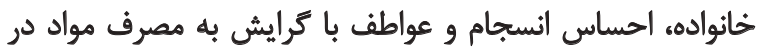

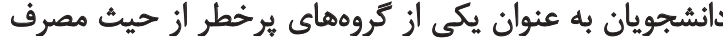

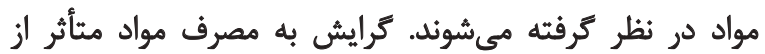

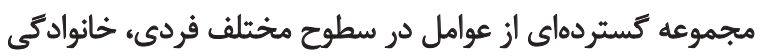

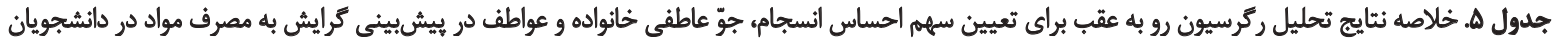

\begin{tabular}{|c|c|c|c|c|}
\hline سطح معنادارى (P) & $\mathbf{t}$ & بثا & B & متغيرهاى ييشبين \\
\hline$<\cdot 1 \cdot \Delta$ & $-r / \cdot 9$ & $-.1 \cdot v$ &.$- / \cdot A r$ & ادراك يذيرى \\
\hline$<+1+1$ & $-r / M q$ &.$- / 18 \mathrm{~V}$ &.$- / l a r$ & معنادارى \\
\hline$<+1 \cdot 1$ & $-r / F r$ &.$- / M A P$ &.$- / 198$ & كتترلئيرى \\
\hline$<+1+1$ & $-1 / 80$ & -.1 .98 &.$- / F A E$ & تأييد كردن \\
\hline$<+1 \cdot 1$ & -r/eg &.$- / 1 F$ &.$- \mid E M A$ & تجريهاي مشترى \\
\hline$<+1+\Delta$ & $-r / \cdot 1$ &.$- / 114$ & $-. / R+4$ & هديه دادن \\
\hline$<+1 \cdot \Delta$ & $-r / T V$ &.$- / / F T$ & - iafa & تشويق كردن \\
\hline$<+1 \cdot \Delta$ & $-r / T H E$ & $-* / 1 F A$ &.$- / 898$ & اعتماد كردن \\
\hline$<+1+\infty$ & $-r / F$ & $-\cdot / N A F$ & -.189. & احساس امثيت \\
\hline$<.1 .1$ & $r / \cdot r$ &.$/ 18$ &. Mef & عاطفئ منفى \\
\hline
\end{tabular}

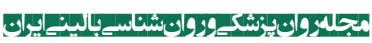


جدول و. نتايج تحليل ركرسيون رو به عقب براي تعيين سهم كلى احساس انسجام، جوّ عاطفى خانواده وعواطف در بيشبينى ترايش به مصرف مواد در دانشجويان

\begin{tabular}{|c|c|c|c|c|}
\hline سطح معنادارى (P) & درجه آزادى & $\mathbf{F}$ & $\mathbf{R}^{r}$ & $\mathbf{R}$ \\
\hline$<+/ .+1$ & $1+-m+1$ & r &.$/ F W$ & .1991 \\
\hline
\end{tabular}

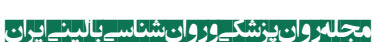

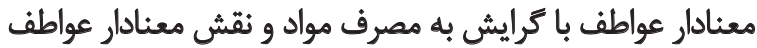

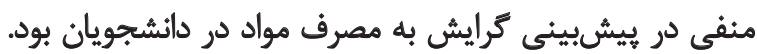

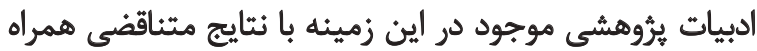

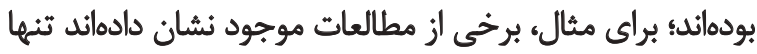

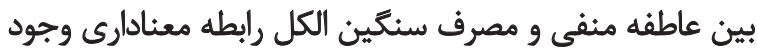

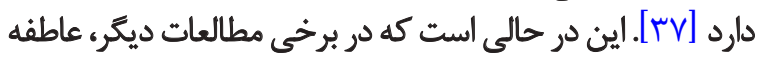

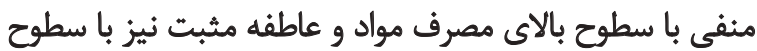

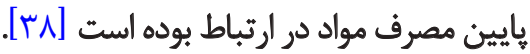

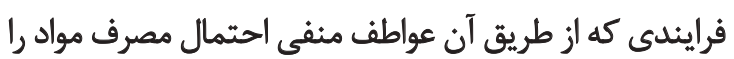

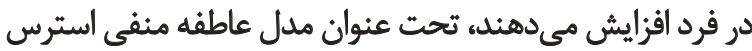

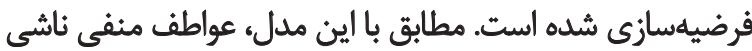

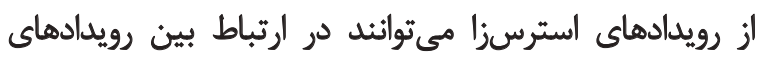

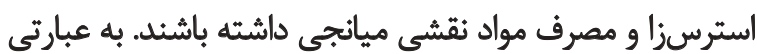

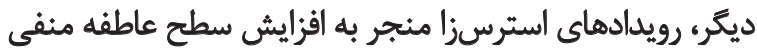

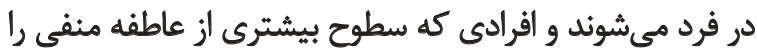

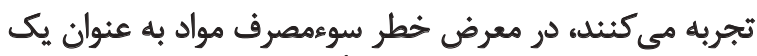

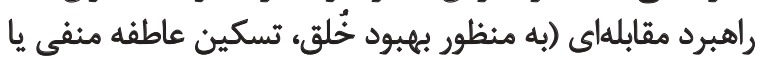
حواسيرتى از احساسات و عواطف ناخوشايئد ناشى از رازئ رويداد

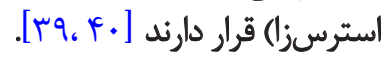

علاوه بر اين، يكى ديكر از ابعاد دخيل در عواطف كه ميثتواند

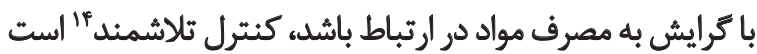

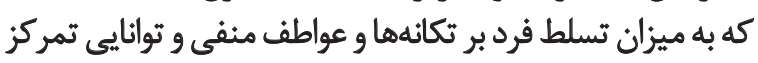

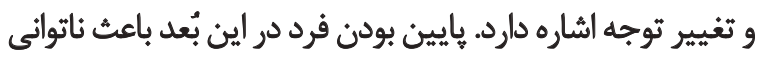

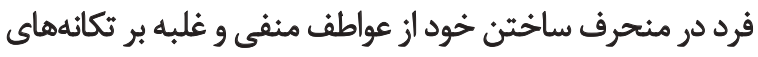

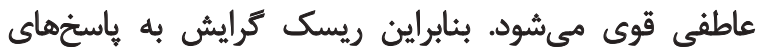

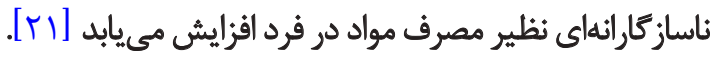

\section{نتيجليَّى}

نتايج اين يُروهش حاكى از ارتباط معنادار بين جوّ عاطفى دوري

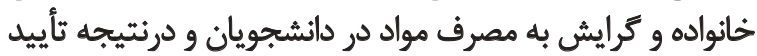

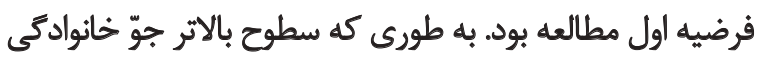

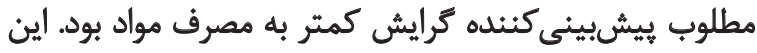

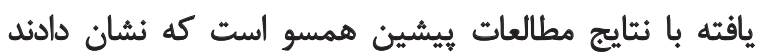

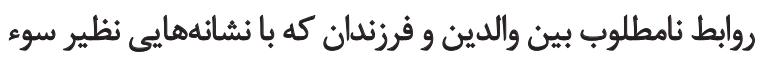

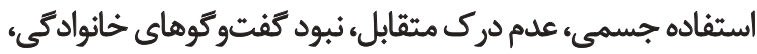

كروهى از دانشجويان بررسى شد.

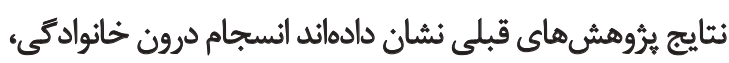

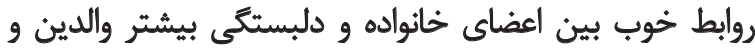

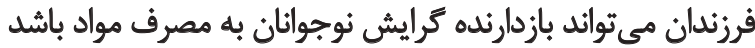

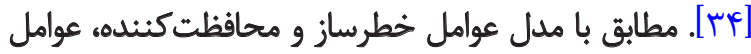

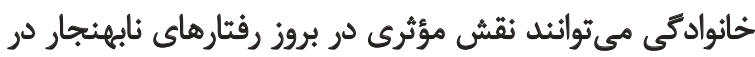

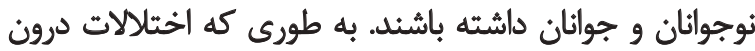

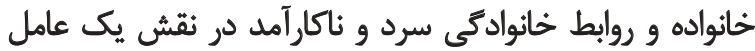

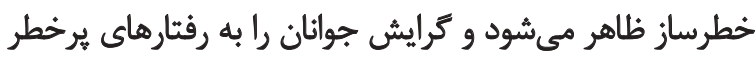

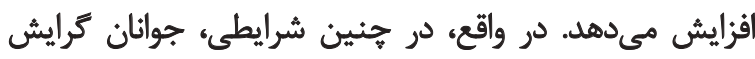

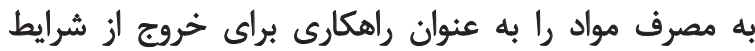

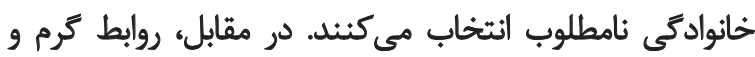

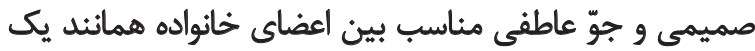

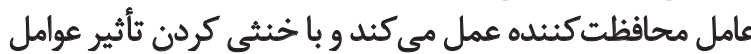

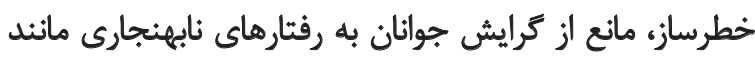

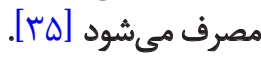
ورخى مطالعات نشان دادهاند انسجام و ارتباطات عاطفى كمتر

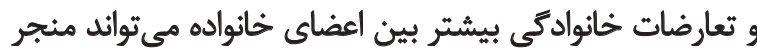

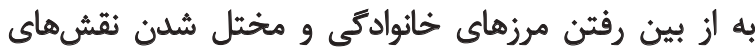

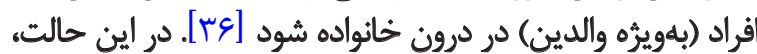

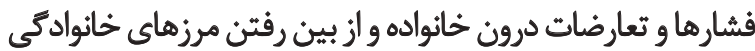

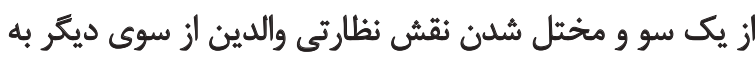

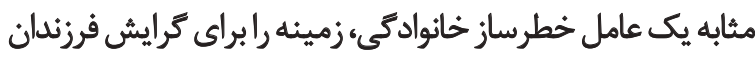

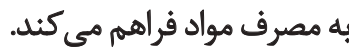

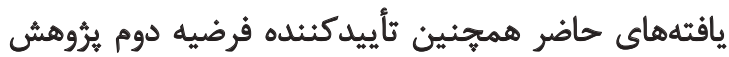

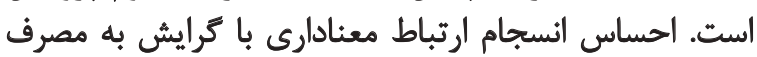

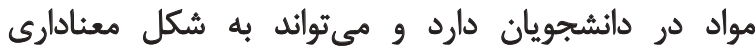

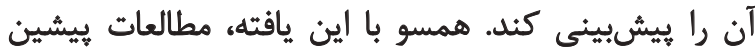

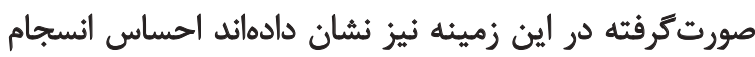

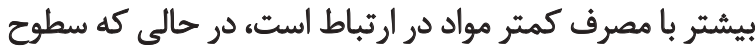

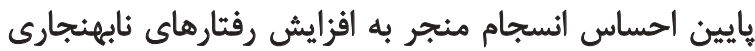

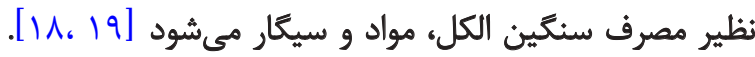

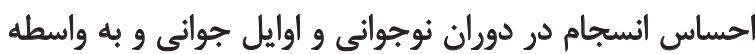

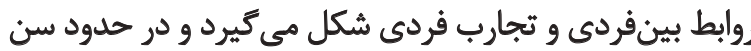

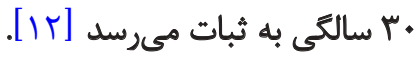

نتايج اين مطالعه نشاندهنده تأييد فرضيه سوم و ارتباط 
كه نمونه مطالعه حاضر از بين دانشجويان دانشعَاه فرهنغيان

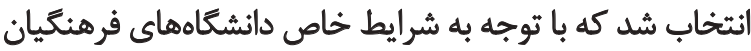

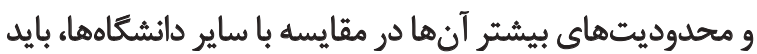
در تعميه يافتهها به ساير دانشجويان احتياط كرد.

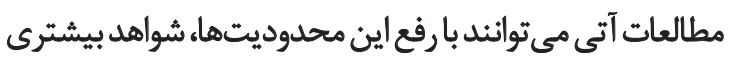

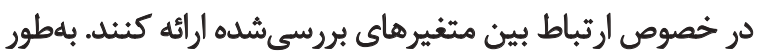

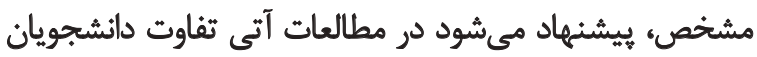
مصرفكننده و غير مصرف كننده مواد (شامل سيعار، الكل و مواد

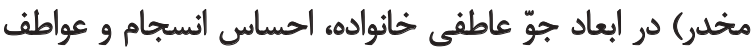

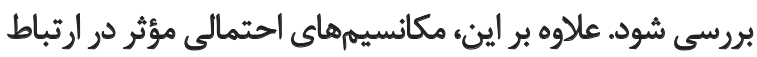

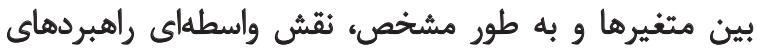

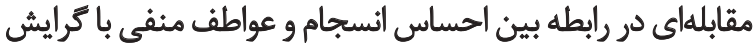

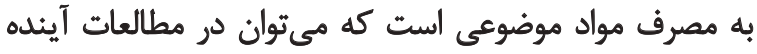

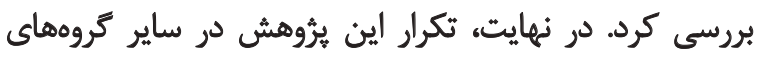

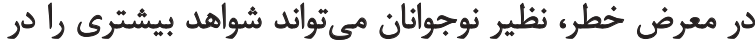

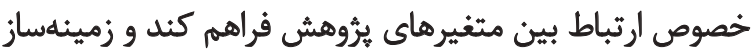

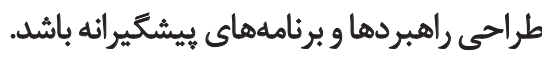

ملاحظات اخلاقى

\section{ي بيروى أز اصول اخلاق بؤوهش}

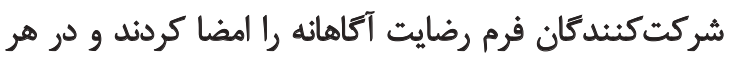

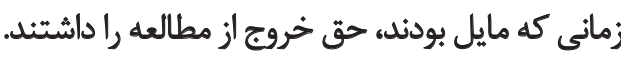

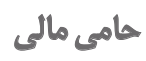

مقاله حاضر از رساله دكترى محمد ارقبايي در كروه

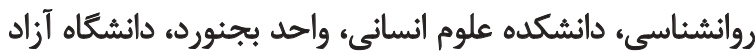

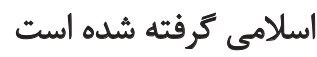

$$
\text { تعارض مثاقع }
$$

بنا به اظهار نويسنده مسئول مقاله، تعارض منافع وجود

نداشُتئه است. به.

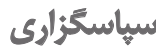

در خايان از تمامى شركت كنندكان محترم كه ما را در تكميل اين يُؤهش يارى كردند، تشكر مى كنيم.

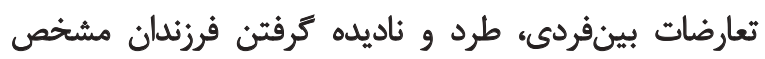

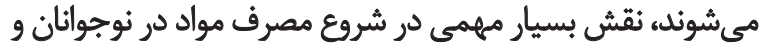

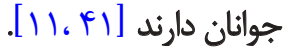

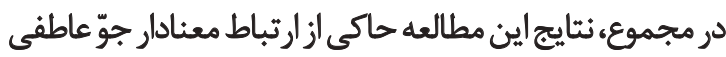

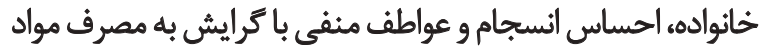

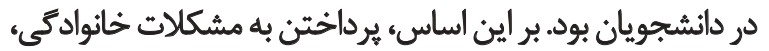

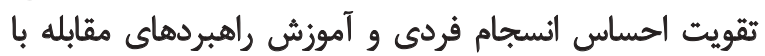

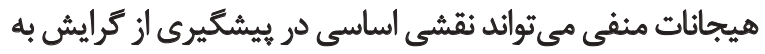
مصرف مواد در دانشجويان داشته باشند

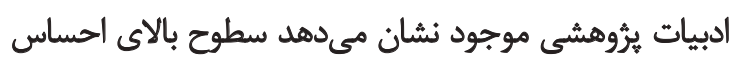

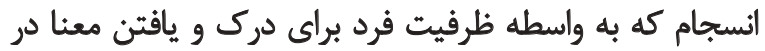

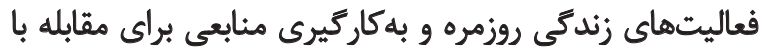

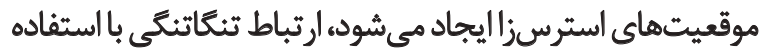

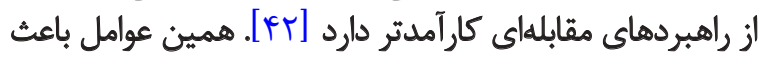

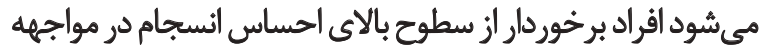

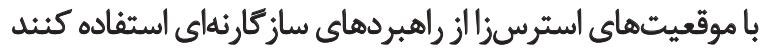

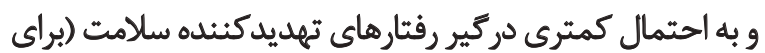

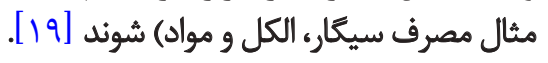
در مقابل، سطوح بايين احساس انسجام بلويره در افراد كمتر

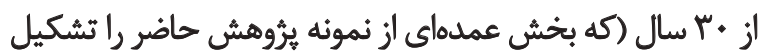

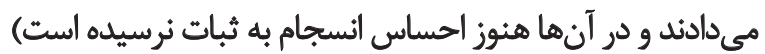

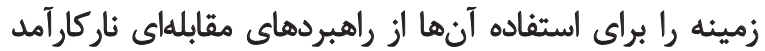

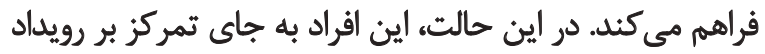

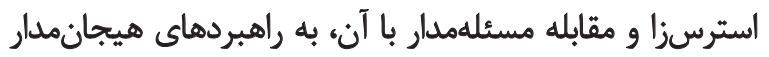

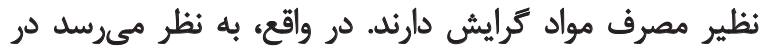

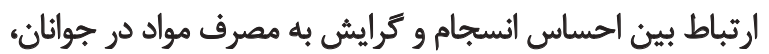

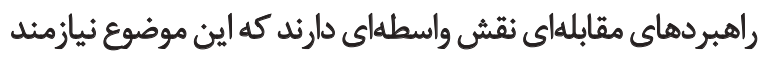
بررسى هاى بيشترى در مطالعات آتى است.

از محدوديتهاى مطالعه حاضر مىثتوان به اين موارد

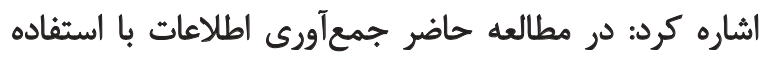

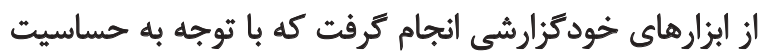

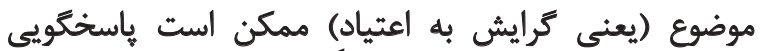

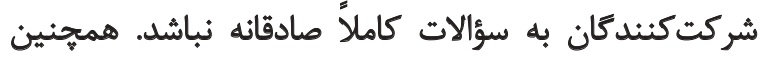

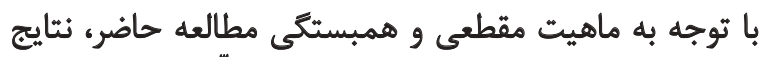

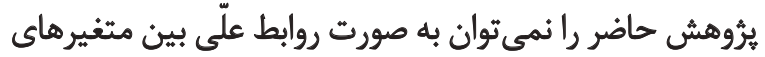

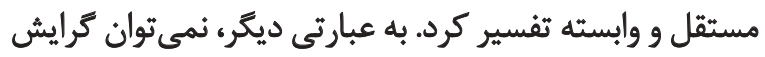

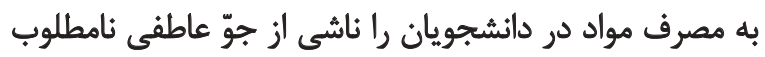

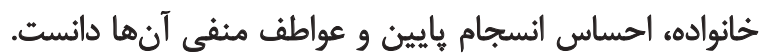

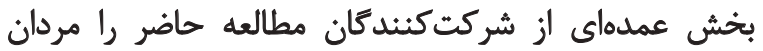

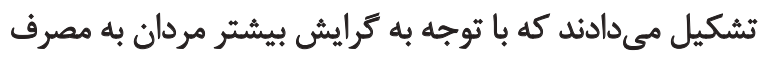

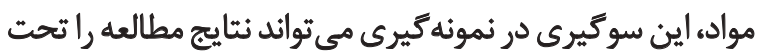

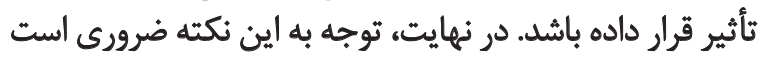




\section{References}

[1] Sahebolzamani M, Alilou L, Rashidi A, Shakibi A. [Determining individual characteristics of addicts through multi-dimensional "MMPI" questionnaire who referred to the treatment centers of Tehran in 2008 (Persian)]. Urmia University of Medical Sciences. 2010; 20(4):290-7.

[2] Self DW, Staley JK. Behavioral neuroscience of drug addiction. Berlin: Springer; 2010. [DOI:10.1007/978-3-642-03001-7]

[3] Mostafaei H, Hosseini M, Jenaabadi H. [The investigation of the relationship between aggression and the addiction potential among high school male students (Persian)]. Journal of Management and Accounting Studies. 2014; 2(1):1-4.

[4] Darharaj M, Habibi M, Kelly AB, Edalatmehr Z, Kazemitabar M. Predisposing personality traits and socio-familial factors of tendency toward substance use among soldiers. Journal of Substance Use. 2017; 22(3):310-6. [DOI:10.1080/1 4659891.2016.1195896

[5] Sadock BJ, Sadock VA, Ruiz, P. Kaplan and Sadock's Synopsis of Psychiatry: Behavioral Sciences/Clinical Psychiatry. New York: Wolters Kluwer; 2014

[6] Michael A, Dawes MD. Clinical manual of adolescent substance abuse treatments, by Kaminer Y (Revision). American Psychiatric Association. 2011; 21(5):498-9.

[7] Abdolmaleki S, Farid A, Habibi-Kaleybar R, Hashemi S, Ghodoosi Nejad A. [Investigation the relationship between family emotional atmosphere and affective control with tendency to addiction (Persian)]. Journal of Family Research. 2017; 12(48):649-62.

[8] Mirzzae M, Nasirzadeh M, Eskami AA, Sharifirad G, Hasanzadeh A. [Influence of family function about youth dependence to synthetic drugs (Persian)]. Iranian Journal of Health Education and Health Promotion. 2013; 1(2):19-30.

[9] Ioannis D, Maria T. Findings from a large-scale empirical research on substance abuse prevention in Greece. Global Journal of Addiction \& Rehabilitation Medicine. 2017; 2(5):1-9. [DOI: 10.19080/GJARM.2017.02.555598]

[10] Matejevic M, Jovanovic D, Lazarevic V. Functionality of family relationships and parenting style in families of adolescents with substance abuse problems. Procedia - Social and Behavioral Sciences. 2014; 128:281-7. [DOI:10.1016/j.sbspro.2014.03.157]

[11] Mirković-Hajdukov M, Spahić TE, Softić R, Bećirović E, Šimić J. Family atmosphere and relationships as predictors of heroin addiction. Psychiatria Danubina. 2017; 29(2):129-33. [PMID]

[12] Antonovsky A. Unraveling the mystery of health: How people manage stress and stay well (Jossey Bass Social and Behavioral Science Series). San Francisco: Psychology \& Counseling; 1987.

[13] Viljoen A. Burnout, coping, and sense of coherence in an engineering organization [MA thesis]. Pretoria: University of South Africa; 2012.

[14] Kouvonen AM, Väänänen A, Vahtera J, Heponiemi T, Koskinen A, Cox SJ, et al. Sense of coherence and psychiatric morbidity: A 19-year register-based prospective study. Journal of Epidemiology and Community Health. 2010; 64(3):255-61. [DOI:10.1136/jech.2008.083352] [PMID]
[15] Binkowska-Bury M, Januszewicz P. Sense of coherence and health-related behavior among university students: A questionnaire survey. Central European Journal of Public Health. 2010; 18(3):145-50. [PMID]

[16] Blom EC, Serlachius E, Larsson JO, Theorell T, Ingvar M. Low sense of coherence is a mirror of general anxiety and persistent depressive symptoms in adolescent girls-a cross-sectional study of a clinical and a non-clinical cohort. Health and Quality of Life Outcomes. 2010; 8(1):58-63. [DOI:10.1186/1477-7525-8-58] [PMID] [PMCID]

[17] Kikuchi Y, Nakaya M, Ikeda M, Okuzumi S, Takeda M, Nish $\mathrm{M}$. Sense of coherence and personality traits related to depressive state. Psychiatry Journal. 2014; 1:1-6. [DOI:10.1155/2014/738923] [PMID] [PMCID]

[18] Grevenstein D, Bluemke M, Nagy E, Wippermann CE, Kroeninger-Jungaberle $\mathrm{H}$. Sense of coherence and substance use: Examining mutual influences. Personality and Individual Differences. 2014; 64:52-7. [DOI:10.1016/j.paid.2014.02.017]

[19] García-Moya I, Jiménez-Iglesias A, Moreno C. Sense of coherence and substance use in Spanish adolescents: Does the effect of SOC depend on patterns of substance use in their peer group? Adicciones. 2013; 25(2):109-17. [DOI:10.20882/adici ciones.58] [PMID]

[20] Yilmaz H, Arslan C. Subjective well-being, positive and negative affect in Turkish university students. Online Journal of Counseling \& Education. 2013; 2(2):1-8.

[21] Cheetham A, Allen NB, Yücel M, Lubman DI. The role of affective dysregulation in drug addiction. Clinical Psychology Review. 2010; 30(6):621-34. [DOI:10.1016/j.cpr.2010.04.005] [PMID]

[22] Tate SR, Patterson KA, Nagel PB, Anderson KG, Brown SA Addiction and stress in adolescents. In: Al'Absi M, editor. Stress and addiction: Biological and psychological mechanisms. San Diego: Academic Press; 2010.

[23] Lee CH, Chang FC, Hsu SD, Chi HY, Huang LJ, Yeh MK. Inappropriate self-medication among adolescents and its association with lower medication literacy and substance use. PLOS One. 2017; 12(12):1-14. [DOI:10.1371/journal.pone.0189199] [PMID] [PMCID]

[24] Ryan F. Cognitive therapy for addiction: Motivation and change. Hoboken, New Jersey: John Wiley \& Sons; 2013. [DOI:10.1002/9781118316474]

[25] Sarmad Z, bazargan A, Hejazi E. [Research methods in behavioral sciences (Persian)]. Tehran: Agah; 2016

[26] Hooman A. [Scientific methods in behavioral sciences (Persian)]. Tehran: SAMT; 2001

[27] Heilbrun AB. Parental model attributes, nurturing reinforcement, and consistency of behavior in adolescents. Child Development. 1964; 35(1):151-67. [DOI:10.2307/1126580] [PMID]

[28] Haghighi J, Shokrkon H, Mousavi M. [The relationship between family emotional atmosphere and female students' adjustments at middle schools in Ahvaz (Persian)]. Journal of Educational Sciences and Psychology. 2002; 3(1):79-108.

[29] Antonovsky A. The Structure and Properties of the Sense of Coherence Scale. Social Science \& Medicine. 1993; 36(6):725-33. [DOI:10.1016/0277-9536(93)90033-Z] 
[30] Alipour A, Nasim S. [Validity and reliability of the Sense of Coherence (SOC) questionnaire in university students (Persian)]. Pajoohandeh Journal. 2012; 17(1):50-56.

[31] Watson D, Clark LA, Tellegen A. Development and validation of brief measures of positive and negative affect: the PANAS scales. Journal of Personality and Social Psychology. 1988; 54(6):1063-70. [DOI:10.1037/0022-3514.54.6.1063] [PMID]

[32] Bakhshipour A, Dezhkam M. Factor analysis of Positive and Negative Affects Scale. The Journal of Psychology. 2006; 9(4):351-65.

[33] Mirhesami S. Examining the role of family in adolescents' and youths' tendency toward addiction [BSc. thesis]. Tehran: Payame Nour University; 2009.

[34] Kopak AM, Chen AC, Haas SA, Gillmore MR. The importance of family factors to protect against substance use related problems among Mexican heritage and White youth. Drug and Alcohol Dependence. 2012; 124(1):34-41. [DOI:10.1016/j.drugalt cdep.2011.12.004] [PMID] [PMCID]

[35] Hawkins JD, Catalano RF, Miller JY. Risk and protective factors for alcohol and other drug problems in adolescence and early adulthood: Implications for substance abuse prevention. Psychol Bull. 1992; 112:64-105. [DOI:10.1037/0033-2909.112.1.64] [PMID]

[36] Schafer G. Family functioning in families with alcohol and other drug addiction. Social Policy Journal of New Zealand. 2011; 37(2):135-51

[37] Brunborg GS. Positive and negative affectivity as risk factors for heavy drinking in the second half of life: A prospective cohort study. Addict. 2017; 112(5):801-7. [DOI:10.1111/ add.13718] [PMID]

[38] Wills TA, Sandy JM, Shinar O, Yaeger A. Contributions of positive and negative affect to adolescent substance use: Test of a bidimensional model in a longitudinal study. Psychology of Addictive Behaviors. 1999; 13(4):327-38. [DOI:10.1037/0893-164X.13.4.327]

[39] Wills TA, Shiffman S. Coping and substance use: A conceptual framework. Orlando: Academic Press; 1985.

[40] Mandavia A, Robinson GG, Bradley B, Ressler KJ, Powers A. Exposure to childhood abuse and later substance use: Indirect effects of emotion dysregulation and exposure to trauma. Journal of Traumatic Stress. 2016; 29(5):422-9. [DOI:10.1002/jts.22131] [PMID] [PMCID]

[41] Bernardy CC, Oliveira ML. The role of family relationships in the initiation of street drug abuse by institutionalized youths. Revista da Escola de Enfermagem da USP. 2010; 44(1):11-17. [DOI:10.1590/S0080-62342010000100002]

[42] Sarenmalm EK, Browall M, Persson LO, FallDickson J, Gaston Johansson F. Relationship of sense of coherence to stressful events, coping strategies, health status, and quality of life in women with breast cancer. Psychooncology. 2013; 22(1):20-7. [DOI:10.1002/ pon.2053] [PMID] 